\title{
Prevalence of Intestinal Nematodes among Children below ten years attending Kiwoko Hospital Pediatric Unit, Kasana, Nakaseke, District, Uganda.
}

\author{
Henry Pecos Nvule ${ }^{a}$ \\ a Faculty of Health Sciences, Uganda Martyrs University, \\ Uganda
}

Abstract

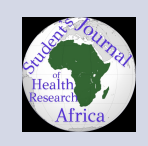

Background: ${ }^{a}$

Intestinal nematodes are among the most common parasites infecting humans in developing countries, precise estimates of the populations at risk of infection are difficult to derive. Therefore to understand the disease burden there is a need for reliable data on the prevalence of infection as well as information on the risk factors of infection.

Methodology:

99 stool samples were collected from children who presented with diarrhea, fever, itchy anus opening all symptoms of intestinal nematode infection. Stool samples were screened using the direct saline method. Samples negative by direct saline were further examined using the formal ether technique and stained by eosin for visualization of parasites eggs. A questionnaire was administered to their guardians/parents to assess the risk factors of infection.

Results:

The prevalence of intestinal nematode infection was found to be $62.6 \%$ (62/99). The intestinal nematode infection rate was found to be higher in children over five years as compared to children below five years with a prevalence of $64.2 \%$ $(43 / 67)$ and $59.4 \%$ (19/32) respectively. The prevalence of infection by sex was $66 \%(31 / 47)$ in males and $59.6 \%(31 / 52)$ in females.

Ascaris lumbricoides were the most prevalent parasite identified in 25 of the 62 patients infected with intestinal nematodes. This was followed by Hookworm (17/62), Trichuris trichiura (12/62), and Strongyloides stercoralis (8/62). Poor hygiene was the most common risk factor cited followed by illiteracy and poverty.

\section{Conclusion and recommendations:}

The results show intestinal nematode infection is common in children attending the Kiwoko pediatric unit. This, therefore, calls for different stakeholders such as public health officers, medical personnel, and the community to develop new strategies to educate the community on existing government programs aimed at the elimination of intestinal nematode infection in children in the Nakaseke district.

\footnotetext{
recieved: 11th/02/2021 accepted:

10th/04/2021 email: nvupec@gmail.com
}

\section{Background}

Intestinal nematode infection is a big burden in developing countries particularly in children living in rural communities (Brooker et al., 2010). Globally, more than one billion people are infected with one or more of the different intestinal nematodes species with $A$. lumbricoides and T. trichura being at 
maximum prevalence in children below ten years, (Risika et al., 2013; Ojja et al., 2018). Intestinal nematodes are globally distributed in the tropics and subtropics regions of the world, particularly in SubSaharan Africa, where parasites present a major public health problem. Intestinal nematodes are a debilitating parasitic disease in many parts of Uganda including the Nakaseke district. Previous studies in the Luweero district indicated that intestinal nematode infection caused $9 \%$ morbidity in children below five years and was ranked third among the top 10 diseases of the district in children under five.

The helminth infections of children consisted of $82.1 \%$ Ancylostoma duodenale or Necator americanus, $18.9 \%$ Ascarislumbricoides, $7.0 \%$ Trichuristrichiura, 1.0\% with Enterobiusvermicularis, and $0.5 \%$ with Hymenolepis nana (Dumba et al., 2008). These parasites are transmitted via the fecal-oral route through of consumption of food or water contaminated with parasite eggs and larvae. The eggs and larvae of these parasites remain dormant but infective for long periods in the environment facilitating their transmission.

An infection with these parasites is associated with, poor hygiene, lack of access to safe water, low pit latrine coverage, low social status, and poverty (Gizaw et al., 2018). Children infected by these parasites commonly suffer intestinal obstruction, anaemia, malnutrition, dysentery, fever, dehydration, vomiting, colitis cognitive and other impairments which may lead to failure to fully develop mentally and physically.

There is a high burden of intestinal nematode infection in developing countries including Uganda. Due to the poor health systems, most cases are undiagnosed and as a result, there is a paucity of information on the prevalence of intestinal nematode infections particularly in children who bear a greater risk of infection. Secondly, the government of Uganda introduced programs to provide free deworming services to children, however, the effectiveness of this program is not known.

This study, therefore, sought to elucidate on the prevalence of intestinal nematodes among children below ten years of age, and access the risk factors associated with infection. Furthermore, this study sought to evaluate the effectiveness of the free deworming project by the government in the Nakaseke district.

\section{Methodology}

\section{Study area}

Kiwoko Hospital is a community-based hospital found in Nakaseke district, Uganda. The Hospital serves the population in Nakaseke and the nearby districts like Nakasongola and Luwero.

Kiwoko Hospital is located approximately 80 kilometers northwest of Kampala, the capital of Uganda. Up to approximately 100 patients per day are attended to at the laboratory.

\section{Study design}

This was a cross-sectional study done from June 2016 to July 2016 at Kiwoko hospital pediatric unit. Children below ten (10) years who presented with clinical signs and symptoms of intestinal nematode infections were recruited with the consent of their caretaker. Their stool samples were collected, examined both macroscopically and microscopically for the presence of intestinal nematodes.

\section{Study Population}

The study included all children below ten (10) years attending Kiwoko Hospital Pediatric unit with consent from their guardians during the study between June and July 2016.

\section{Inclusion criteria}

Children below 10 years attending pediatric unit presenting with clinical signs and symptoms of intestinal nematode infections like stomach upsets diarrheic stool, fever, and itchy anal opening whose guardians consented were enrolled for the study. Children that did not present with clinical signs and symptoms of intestinal nematode infection as well as those whose guardians did not consent were excluded from this study.

\section{Ethical considerations}

Authorization was obtained from the Medical Director Kiwoko Hospital and the In-charge Pediatric unit and laboratory in charge before the study commenced. Only patients whose guardians had consented were included in the study. To ensure confidentiality, volunteer's initials and laboratory numbers were used and not their names, and their results were known only to the technician and the clinician for appropriate medical intervention.

\section{Sample size}

The sample size was determined using the following formula; $n=4 P Q / E^{2}$ since the study was qualitative and aimed at estimating proportions according to Mahajan (2010)

Where $\mathrm{n}=$ sample size. 
$E=$ allowable error $-10 \%$

$\mathrm{P}=$ prevalence from literature- $54.8 \% . \mathrm{Q}=1-\mathrm{P}$.

$8 \%$ was the prevalence used based on a study by Mahajan (2010). Therefore;

$\mathrm{N}=4 * 0.548 * 0.452 /(0.1)^{2}$

$\mathrm{N}=99$.

Therefore, 99 patients were enrolled into the study.

\section{Sample collection}

Demographic data was collected from the eligible participants using a data collection form. The participant was given a well-labeled sterile, dry, leak-proof container and requested to provide an adequate amount of stool, not contaminated with urine. The time of collection was noted on the specimen container as soon as the volunteer returned the specimen.

For all the stool samples, the macroscopic examination was done and then followed by microscopic examinations using the direct saline and $10 \%$ formol ether concentration technique.

The parasites found were counted and results reported as follows;

- Number of parasites counted species, stage per low power objectives e.g. 2 eggs of

Tirichuris trichura seen/low power field (LPF).

\section{Laboratory procedures}

The samples were recorded into the laboratory register and assigned a laboratory number; the samples were then examined macroscopically and microscopically.

\section{Macroscopic examination}

Stool samples were analyzed for color, consistency, and whether they contained blood, mucus, pus as well as for the presence of macroscopic worms.

\section{Microscopic examination}

\section{Direct saline and eosin (wet) preparation}

A drop of fresh physiological saline was placed at the center of the glass. Using an applicator stick, a small amount of specimen is placed onto the saline and mixed. The sample was then examined using the $x 10$ objective. If intestinal nematode eggs were observed a drop of eosin was placed on the edges of the cover slip to aid identification of the parasite.

\section{$10 \%$ Formol ether concentration technique}

$10 \mathrm{ml}$ of formalin was added to approximately $1 \mathrm{~g}$.of feces and the mixture stirred using an applicator stick until a homogeneous suspension was attained. A paper filter was placed into a funnel and the funnel was placed on top of the centrifuge tube.
The fecal suspension was then passed through the filter into the centrifuge tube until the $7 \mathrm{ml}$ mark was reached. $3 \mathrm{ml}$ of ether was added to the sample and mixed for 1 minute, then centrifuged at $3000 \mathrm{rpm}$ for 1 minute. Using the applicator stick, debris was loosened and the supernatant poured quickly by inverting the tube. The sediment was mixed thoroughly and a smear prepared and an eosin preparation made for examination.

The parasites found were counted and results reported as; several parasites counted, species per low power objectives e.g., 2 eggs of Tirichuris trichura seen/LPF.

Any other findings such as red blood cells, fat globules, and other intestinal parasites were also reported only for the benefit of the patient's management by the clinician.

\section{Data collection tool}

A questionnaire was used as a data collection tool. It comprised of three parts; sociodemographic status, lifestyles, and laboratory investigations. It was written in English comprising both open-ended and close-ended questions

\section{Data management and analysis}

Data was appropriately managed by the researcher to ensure confidentiality and security. Data management also involved cross-checked to ensure that all questions were properly answered before the participants left the study area.

Data obtained from the study were analyzed using Microsoft word, Microsoft excel, and Statistical Package for Social Sciences (SPSS).

\section{Quality Control}

Quality control was ensured through following standard operating procedures in the laboratory for all the tests that were done,

Reagents that were used were checked adequately using known positive and negative controls for their performance.

Reference charts for the egg and larvae of the Intestinal Nematodes were used in the laboratory during microscope examination and guidance from the supervisor was sought throughout the study.

\section{Limitations of the study}

Financial costs led to failure to administer questionnaires to all the participants of the study. Some patients were not willing to co-operate during the study resulting in delays in reaching the required study population 


\section{RESULTS}

\section{Prevalence of intestinal nematodes.}

99 children were sampled in this study, out of the 99 children, 47 were male, and 52 female. 62 children were found to be infected with intestinal nematodes giving a prevalence of $62.6 \%$ as shown in table 1.

\section{Table 1}

Out of the 62 positive patients, 31 were female, and 31 male. $12(12 / 31)$ female children between the ages of 1-5 were infected and 7(7/31) males infected with intestinal nematode parasites giving a percentage of $22.4 \%$. 24 (24/31) male children between the ages of $5-10$ had the parasite infection as well as the $19(19 / 31)$ females as shown in table 2.

Table 2: The table below represents the number of male and females according to the different age groups

Out of the 62 children infected, 39.9\% (25/62) had $A$. lumbricoide infection.

\section{Table 3: Distribution of the} various intestinal nematode infections in infected children

\section{Predisposing factors to Intestinal Nematode} Infections

Compromised Hygiene was the most predisposing factor to intestinal nematodes infection followed by illiteracy, poverty, and lastly education. I discovered that the guardians lacked knowledge about good hygiene practices hence leading to poor hygiene in their communities. A combination of more than one factor was also seen in children through interaction with their guardians. Out of the 99 patients received, 37 had a history of poor hygiene, 15 were illiterate and were aware of the government deworming programs, base on the data presented from the questionnaires, 10 of the patients were poor with. Poor Handwashing habits in children below the age of 10 were associated with intestinal nematodes as shown in table 4.

\section{Table4: Predisposing factors to intestinal nematode infection}

\section{Discussion:}

This study aimed to determine the common intestinal nematode infection in children below 10 years at Kiwoko hospital pediatric unit, to assess awareness and use of free deworming programs in the Nakaseke district. Lastly to determine risk factors for an intestinal infection in the Nakaseke district.

A total of 99 children below the age of 10 years were recruited with consent from their parents. $52(52.6 \%)$ of the respondents were females with the males accounting for 47 (47.5\%). The overall prevalence of nematode infection in children with diarrhea was $62.2 \%$ which should an increase from the previous research done by Kabatereine et al., (2011) which was 55.9\% in children. The most prevalent infection which was still seen in the study that was carried out in Kenya among pre-school going children in 2015 that showed a prevalence of $19.8 \%$ by Nyakang'o et al., (2015), this was followed by $27.15 \%(17 / 62)$ of hookworm infection, $19.16 \%$ $(12 / 62)$ had T. stercolaris and $12.8 \%(8 / 62)$ S. stercolaris infection with the least prevalent parasite species as this is similar with a study done by international intention by Awoyeni et al., (2015) that had a prevalence of $0.5 \%$ hence there was an increase as compared to the previous study as seen in table 3.

Kiwoko hospital is situated in a rural area with no clean water supply to the community, this also attributes to a high prevalence as the population is at risk of using infected water for human consumption. This was seen in a journal of health that showed diarrhea prevalence of $14.4 \%$ done by Blaise et al., (2010).

Secondly, the study was carried out during the dry season with high humidity which increases the hatchability of the geohelminthes thus a high level of infection, as noted in (Chessbrough 2006).

More males were infected with $66 \%(31 / 47)$ as compared to females with $59.6 \%$ (31/52), this came to an agreement with aa study by Nyakang'o et al., (2015) that showed a prevalence of $35.80 \%$ in Kenya.

The males were more infected than females; this disproportion could be due to behavioral and environmental because male children tend to be more 
Number of patients sampled Number of positive patients Prevalence 99 62 $62.6 \%$

Table 1. number of male and females according to the different age groups

\begin{tabular}{llll}
\hline Age & Infected Male & Infected Female & Total \\
$1-5$ & 7 & 12 & 19 \\
$5-10$ & 24 & 19 & 43 \\
Total & 31 & 31 & 62 \\
\hline
\end{tabular}

\begin{tabular}{lllllll}
\hline Sex & No. of participants & A.lumbricoides & Hookworm & T.trichura & S.stercolaris & Total \\
Male & 47 & 10 & 9 & 0 & 6 & $\mathbf{2 5}$ \\
Female & 52 & 15 & 8 & 12 & 2 & $\mathbf{3 7}$ \\
Total & 99 & $\mathbf{2 5}$ & $\mathbf{1 7}$ & $\mathbf{1 2}$ & $\mathbf{8}$ & $\mathbf{6 2}$ \\
\hline
\end{tabular}

\begin{tabular}{ll}
\hline Factor & \\
Poor Hygiene & 37 \\
Illiteracy & 15 \\
Poverty & 10 \\
Education & 7 \\
More than one factor & 30 \\
\hline
\end{tabular}

active in the infected environment without adult supervision as compared to their female counterparts.

The age group of 5-10 (67) had more respondents as compared to the $1-5$ age groups. This could due to the fact children over 5 years communicate with their guardians. Secondly, children over 5 years are of school-going age and are more exposed to the environment contaminated with parasite eggs and cannot follow proper hygiene practices without the supervision of elders hence become infected with intestinal nematodes. 6-10 are school-going and thus increasing the rate of transmission through eating unwashed fruits, and unwashed hands.

A. lumbricoide infection was the most prevalent infection because the area food and drinking water aren't safe due to exposure to human fecal matter, secondly is one of the most common helminthic human infections worldwide

Hookworm infection was second prevalent and this is indicative of lack of footwear among the children the people are poor and children walk barefooted. It is also indicative of the unsanitary fecal disposal methods of the population.
The results obtained are attributed to the presence of the predisposing factors as discussed above. Also, agriculture is the major economic activity e.g., pineapple growing and since the community still uses the manual methods of farming, they stand a risk of hookworm and S. stercolaris penetrating their skin thus leading to an infection.

Therefore, in light of the results from this study, the government must develop policies to ensure compulsory deworming of children who visit health centers in addition to the screening of intestinal nematodes. This will elucidate the disease burden and will inform in the development of policies aimed at eradication of intestinal nematode infection especially in children who bear the greatest burden of disease.

\section{Recommendation}

Putting Community outreaches educating people about the presence of intestinal nematode and their route of transmission.

There should improvement in the awareness of free government deworming programs in the communities for pre-school and school-going children 
for control of infection in the community as well as prevention of re-infection.

Government should provide safe water for use in the communities and improve on its programs of the free deworming campaign and infection interventions to eradicate intestinal nematode.

\section{Acknowledgment}

I would like to thank Kisubi brothers University College management, my supervisor Sarah Gift Witto for guidance they have given to me during this research project.

I would like to thank the entire community of Kiwoko Hospital for the research opportunity. Secondly I would like to thank Mr. Enoch Muwanga the laboratory incharge for the guidance and support during this research project.

\section{References:}

1) Awoyeni E.A., Olaniran O., Hassan-Olajokun R.E., Adewuyi I.K., March, (2015). Prevalence of Intestinal Nematodes among Children in a Secondary Health Care Delivery in Osogbo, Nigeria. International invention journal of medicine and medical sciences (ISS: 2408-7246). Vol. 2(3) pp. 40-43

2) Brooker S., (2010). Estimating the global distribution and disease burden of intestinal nematode infections: adding up the numbers a review. Cambridge University Press Cambridge. https://doi.org/ 10.1016/j.ijpara.2010.04.004

3) Chessbrough M., (2006). District Laboratory Practice for Tropical countries Part 2. https://doi.or g/10.1017/CBO9780511543470

4) Dumba, R., Kaddu, J. B., \& Wabwire Mangen, F. (2008). Intestinal helminths in Luweero district, Uganda. African health sciences, 8(2), 90-96.

5) Gizaw, Z., Adane, T., Azanaw, J. et al. Childhood intestinal parasitic infection and sanitation predictors in rural Dembiya, northwest Ethiopia. Environ Health Prev Med 23, 26 (2018). https://doi.org/10.1 186/s12199-018-0714-3

6) H.Blaise Nguendo Yongs., (2010). Suffering for Water, Suffering from Water: Access to Drinkingwater and Associated Health Risks in Cameroon, The Journal of Health, Population and Nutrition; 28(5): 424-43 https://doi.org/10.3329/jhpn.v28i5.61 50

7) Kabatereine N.B.,Tukahebwa E.M., Brooker S., Alderman H., and Hall A., (2001). Epidemiology of Intestinal Helminth Infestations among School
Children in Southern Uganda. East African Medical Journal 78(6): 283-286. https://doi.org/10.4314/ea mj.v78i6.9019

8)Nyakango L.N., Shivariro R.S., Muleke C. I., and Mokaa D. O., (2015). soil transmitted helminths, prevalence and pre-diposing factors among preschool age children in elburgon municipality,kenya. African journal of science and research, (5) 6: 01-04 (ISSN: 2306-5877)

9) Ojja, S., Kisaka, S., Ediau, M. (2018). Prevalence, intensity and factors associated with soiltransmitted helminths infections among preschoolage children in Hoima district, rural western Uganda. BMC Infect Dis 18, 408 . https://doi.or g/10.1186/s12879-018-3289-0

10). Risika Sowole., Ayoade Adegbite., Rotimi Sowemimo and Edun B. T., (2013). The prevalence of intestinal nematodes and socio-economic implications: a case study among the pupils of muslim public primary school ilese-ijebuogun state south western Nigeria.Ogun State College Of Health Technology Ilese-ljebu P.M.B 2081 ljebu-Ode Nigeria.

\section{list of abbreviations.}


Table 2. List of Abbreviations

\begin{tabular}{ll}
\hline Aamericanus: & Necatoramericanus \\
A duodenale: & Ancylostomaduodenale \\
A lumbriciodes: & Ascarislumbriciodes \\
CD4: & Clusters of differentiation four \\
DNA: & Deoxyribonucleic acid \\
EIA: & Enzyme Immunoassay \\
ELISA: & Enzyme-linked immunosorbent assay \\
GI: & Gastro Intestinal \\
Ig: & Immunoglobulin \\
IL: & Interleukin \\
INI: & Intestinal Nematode Infections \\
Ltd: & Limited \\
PCR: & Polymerase Chain Reaction \\
S. stercoralis: & Strongyloidesstercoralis \\
T. trichiura: & Trichuristrichiura \\
UNICEF: & United Nations International Children's Emergency Fund \\
WHO: & World Health Organization \\
\hline
\end{tabular}

